\title{
Explaining the association between subjective social status and mental health among university students using an impact ratings approach
}

\section{Mark Rubin ${ }^{1}$}

Received: 3 June 2020 / Accepted: 31 October 2020 / Published online: 10 December 2020

(c) Springer Nature Switzerland AG 2020

\begin{abstract}
Previous research has found a positive association between social class and mental health among university students. Various mediators of this association have been proposed. However, the extent to which students perceive these mediators as having an impact on their mental health has not been investigated. It is important to investigate this issue because students who do not perceive issues as having an impact on their mental health may not be motivated to address those issues. In the current study, 402 first-year undergraduate psychology students from a large Australian university indicated the extent to which 32 issues had a negative impact on their mental health over the past six months. Students rated lack of money, time management, coursework assessment items, lack of sleep, and course marks as having the largest impact on their mental health. Lack of money and time management mediated the positive association between subjective social status and mental health over (a) the past week and (b) the past month. Coursework assessment items and course marks mediated the positive association between subjective social status and satisfaction with the university experience. It is concluded that interventions should focus on these relatively high impact issues in order to address social class differences in students' mental health and university satisfaction.
\end{abstract}

Keywords Course assessment · Lack of money $\cdot$ Mental health $\cdot$ Social class · University satisfaction · University students

Mark Rubin

Mark.Rubin@newcastle.edu.au

1 School of Psychology, Behavioural Sciences Building, The University of Newcastle, Callaghan, NSW 2308, Australia 


\section{Introduction}

Students from lower socioeconomic (SES) backgrounds tend to have poorer mental health at university than students from higher SES backgrounds (Ibrahim et al., 2013; King et al. 2011; Rubin et al. 2016; Rubin and Kelly 2015; Said et al. 2013; for a recent review, see Rubin et al. 2019). Prior work in this area has found that classism, sense of control, social contact with other students, and parenting style operate as mediators (Allan et al. 2016; Ibrahim et al. 2013; Rubin et al. 2016; Rubin and Kelly 2015). In other words, controlling for these variables tends to reduce the size of the association between social class and mental health, suggesting that social class differences in mental health are partly explained by social class differences in classism, sense of control, social contact, and parenting style. However, it remains unclear whether students perceive these sorts of issues as having a substantial impact on their mental health. No prior research has addressed this question of perceived impact, and yet there are two reasons why it should be addressed.

First, from a theoretical perspective, students may have informed and useful views on the extent to which external factors impact on their mental health (Juniper et al. 2012). Certainly, students may be biased in these views and potentially in error. Nonetheless, they believe that they know what has been "stressing them out," and these beliefs may correspond with reality. Hence, asking students to rate the impact of potential stressors on their mental health may allow researchers to identify stressors that have an objectively high impact on mental health.

Second, from an applied perspective, students who perceive a link between an issue and their mental health may be more motivated to address that issue in any social intervention that is designed to improve their mental health. For example, a lack of social contact with other students may mediate the positive association between social class and mental health (Rubin et al. 2016). However, interventions that aim to reduce social class differences in social contact in order to improve mental health may not be effective if students do not buy into the idea that improved social contact will reduce their mental health problems. Hence, understanding which potential mediators are perceived to be impactful by students is important in the case of interventions that require active participation and endorsement by students.

Juniper et al. (2012) conducted an impact analysis study with $\mathrm{PhD}$ students. In this study, 1202 students indicated (a) whether they had experienced a series of work-related issues over the past six months and (b) how important each issue was to their well-being. The researchers then computed an impact score, which was the product of (a) the frequency with which students had experienced an issue and (b) the perceived impact of that issue on their well-being. In contrast to Juniper et al.'s study, the present study sampled 1st-year psychology undergraduate students rather than $\mathrm{PhD}$ students. In addition, instead of assessing experience and importance separately and then combining them to form an impact score, the present study asked students to rate the "negative impact" of a series of issues on their mental health over the past six months using a single scale. Hence, in the 
present study, experience and importance were measured together as part of an overall assessment of impact. Similar to Juniper et al., these impact ratings were then ranked in order to identify high impact issues.

It is also important to note that Juniper et al. (2012) did not measure either social class or general mental health. Hence, Juniper et al.'s research was unable to either assess or explain the association between social class and mental health. In contrast, in the current project, mediators of the association between subjective social status and mental health were identified from among the group of high impact issues. Hence, unlike Juniper et al.'s study, the present research was focused on identifying high impact mediators of the association between subjective social status and mental health.

In summary, the current study aimed to provide a better understanding of (a) issues that undergraduate students perceive to be particularly detrimental to their mental health and (b) high impact issues that help to explain the positive association between subjective social status and mental health. This approach allowed us to be more confident that social interventions based on the identified mediators are more likely to be accepted by the students in question.

\section{Method}

\section{Procedure}

This study was approved by the administering university's human ethics research committee. The study was also preregistered. The study and final analyses deviated from the preregistered protocol in several ways. These deviations are identified in an updated preregistration document, which is available at the link indicated in Data Availability statement.

Participants were sampled from a university in New South Wales, Australia. The university had around 37,500 students, and around $27 \%$ of these were classed as low socioeconomic status (SES) students based on their residence in low SES locations.

Data collection occurred between 16th July 2019 and 22nd April 2020. This period included all of Semester 2, 2019 and the first part of Semester 1, 2020. The survey was titled "What's Stressing You Out?," and it was introduced as "investigating the factors associated with your mental health." Participants were sampled from three first-year undergraduate psychology courses. They completed the survey anonymously, in their own time, and via an online survey delivery platform. They received course credit in exchange for their participation.

The order of presentation of measures, and the items within those measures, was randomized for each participant. The exceptions were that the demographic items and perceived awareness of the research hypotheses items (Rubin 2016) were presented at the end of the survey, with the demographic variables presented in a single fixed order.

Participants completed an informed consent item at the end of the survey after they had been made fully aware of all of the items in the survey and provided their 
responses to those items. Participants took a median time of $8.98 \mathrm{~min}$ to complete the survey.

\section{Sample size}

The raw data contained 428 responses. Of these, four participants completed the survey twice. In these cases, the first response was retained, and the second response was deleted. Hence, the total number of participants who completed the survey was 424. Of these, six participants dropped out partway through the survey and did not complete the informed consent item at the end of the survey. A further 10 participants completed the survey but actively declined their informed consent for their data to be included in the data analysis. After deleting these 16 participants, there were 408 participants. Finally, six participants were excluded from the analyses because they indicated that they had English language difficulties in understanding some parts of the survey. Hence, the final number of participants who were included in the data analysis was 402 .

\section{Sensitivity analysis}

Using Faul et al.'s (2007) G*Power 3 program, a sensitivity analysis found that a correlation analysis using a sample size of 402, a power level of 0.85 , and a twotailed alpha level of 0.05 is able to detect an effect size of $r=0.15$, which is a "small" to "medium" sized effect in psychology (Funder and Ozer 2019). This effect size was considered acceptable given that prior research using similar measures and populations to the current research has found that the association between subjective social status and mental health is around $r=0.18$ (Rubin et al. 2016; see also Lorant et al. 2003). In addition, Schoemann et al.'s (2017) Monte Carlo power analysis for indirect effects found a power of 0.82 to detect a mediation effect using a sample size of 402 when the correlations between the predictor, mediator, and outcome are all $r=0.18$.

\section{Measures}

\section{Predictor variable: subjective social status}

Subjective social status was measured together with the other demographic variables using an adapted version of the MacArthur Subjective Social Status scale (Adler et al. 2000). The strengths of this measure are that it is a brief, intuitive, face-valid scale that has been shown to have good convergent validity with objective measures of social class and good predictive validity in relation to physical health and social behaviour (Adler et al. 2000; for reviews, see Diemer et al. 2013, pp. 104-105; Kraus et al. 2011). In addition, subjective measures such as the MacArthur scale have been recommended for use in higher education settings, where students' objective levels of social class (e.g., education level, occupational status, income) are less diagnostic and/or applicable (Diemer et al. 2013; Rubin et al. 2014). I adapted the 
MacArthur scale by removing the associated ladder diagram, because it was difficult to operationalize this diagram in the online survey. Instead, participants moved a slider on a 101-point scale to indicate where they would place themselves relative to other people in Australia in terms of their income, education, and occupation. The sliding scale was anchored lowest income, education, and occupation (0) and highest income, education, and occupation (100). The scale scores were normally distributed in the present sample (skewness $=-0.26$, kurtosis $=-0.78$ ). In addition, prior research has shown that this adapted version of the MacArthur scale has good convergent validity with self-report measures of parental education, prestige of parental occupation, and family wealth among university students (Rubin and Stuart 2018, p. 240). Please note that the MacArthur scale is a continuous measure of subjective social status, rather than a categorical measure of social class. Hence, the terms "lower subjective social status" and "higher subjective social status" are relative to students who report having an average social status score on this measure. In the present sample, the mean subjective social status score was $51.11(S D=19.31)$.

\section{Prospective mediator variables: perceived impacting issues}

Participants were asked to indicate the extent to which they felt that each of 32 issues had a negative impact on their mental health over the past six months. Table 1 lists these 32 issues. The issues were sourced from an informal review of the mental health and university student literature that was conducted by myself. I selected each issue on the basis that (a) it had the potential, in theory, to affect mental health, and (b) it was relevant to university students. I acknowledge that I may have omitted some potential issues in this list, and that future research may adopt a more systematic approach to this matter (e.g., Juniper et al. 2012). Nonetheless, I believe that I have represented the key issues that may affect students' mental health.

Participants provided their responses to each issue on a 101-point sliding scale anchored no impact on my mental health (0), a moderate impact on my mental health (50), and a very large impact on my mental health (100). Note that it is important to interpret the scores on this scale correctly. A low score indicates that the student has either not experienced an issue or they have experienced an issue but it has not affected their mental health. In contrast, a high score indicates that a student has both experienced an issue and that it has affected their mental health.

\section{Outcome variable: mental health}

Participants completed the 21-item Depression Anxiety Stress Scale (DASS; Henry and Crawford 2005). The DASS consists of three 7-item subscales that assess depressive symptoms, anxiety, and stress over the past week. Example items are "I felt that life was meaningless" (depressive symptoms), "I felt scared without any good reason" (anxiety), and "I found it difficult to relax" (stress). Participants responded to items using a 4-point scale anchored never (0) and almost always (3).

Participants also completed the 5-item Brief Mental Health Inventory (BMHI; Berwick et al. 1991). The BMHI assesses psychological well-being and distress (depression and anxiety) over the past month, and it is suitable for non-clinical 
Table 1 List of prospective mediator variables

\author{
Alcohol use \\ Attending classes (lectures, tutorials, labs, etc.) \\ Being bullied \\ Course marks \\ Coursework assessment items \\ Difficulty understanding course material \\ Drug use \\ Eating disorder \\ Family members \\ Finding a job after university \\ Formal examinations \\ Grief \\ Homesickness \\ Lack of exercise \\ Lack of money \\ Lack of sense of belonging at university \\ Lack of sleep \\ Loneliness \\ Managing childcare \\ Non-university work \\ Not knowing how things work at university \\ Other students \\ Physical health \\ Prejudice and discrimination against me \\ Romantic relationships \\ Sexual abuse/harassment \\ Sexual identity \\ Time management \\ Travelling to and from university \\ Unhealthy diet (e.g., fast food, no fruit and veg) \\ University staff members (lecturers, tutors, etc.) \\ Victim of violence
}

populations. The initial part of the scale asks: "How much of the time, during the last month, have you..." and example items are "been a very nervous person?" and "felt downhearted and blue?" Participants responded using a 6-point scale anchored never (1) and all the time (6).

Finally, participants reported changes in their mental health since starting university. Participants responded using a 201-point sliding scale anchored got much worse (-100), stayed the same (0), and got much better (100). 


\section{Control variables}

Previous research has shown that students from lower SES backgrounds tend to be less satisfied than students from higher SES backgrounds with their university experience (Martin 2012). In addition, satisfaction with university is positively related to students' mental health (e.g., Almhdawi et al. 2018; Karaca et al. 2019). Hence, it may be useful to control for satisfaction at university when investigating the association between subjective social status and mental health. Participants completed a 4-item ad hoc measure of satisfaction with their university experience. Example items are "I am satisfied with my university experience," and "I am having a difficult time at university" (reverse scored). Participants responded using a 9-point scale anchored very strongly disagree (1) and very strongly agree (9).

Participants also completed a 4-item ad hoc measure of intention to remain at university. Example items are "I am confident that I will be able to complete my university degree," and "I don't want to carry on with my university degree" (reverse scored). Participants responded using the same scale as that used for the measure of satisfaction with university.

Finally, it is important to appreciate that participants in the current study (a) indicated the extent to which various issues impacted on their mental health and (b) responded to mental health scales. Hence, it is possible that participants believed that their responses should be consistent between these measures, and that they conformed to this implicit demand characteristic in order to please the researcher. To address this issue, the Perceived Awareness of the Research Hypothesis (PARH) scale was included in the survey. This 4-item scale measures the extent to which participants believe that they are aware of the research hypotheses (Rubin 2016). An example item is "I knew what the researchers were investigating in this research." Participants responded on a 7-point scale anchored strongly disagree (1) and strongly agree (7).

\section{Demographic variables}

Participants completed the following 14 demographic items: gender, age, ethnic background, sexual orientation, time of commencement at university, enrolment status (full-time/part-time), mode of study (on campus/distance), campus of enrolment, faculty of enrolment, whether English is first language, course of study, difficulty understanding the survey, whether they had ever been diagnosed by a doctor as having a mental illness (yes/no/prefer not to say), and help-seeking from a mental health professional.

\section{Results}

\section{Sample characteristics}

Participants had a mean age of 23.91 years $(S D=8.51)$, ranging from 17 to 60 years. Table 2 provides a breakdown of the categorical demographic and other variables. 
Table 2 Demographic and other variables

\begin{tabular}{|c|c|c|}
\hline Variable & $N$ & Percentage \\
\hline \multicolumn{3}{|l|}{ Gender } \\
\hline Women & 337 & $83.83 \%$ \\
\hline Men & 63 & $15.67 \%$ \\
\hline Other & 2 & $0.50 \%$ \\
\hline \multicolumn{3}{|l|}{ Ethnicity } \\
\hline White & 347 & $86.32 \%$ \\
\hline Aboriginal & 15 & $3.73 \%$ \\
\hline Asian & 14 & $3.48 \%$ \\
\hline Other & 14 & $3.48 \%$ \\
\hline Prefer not to say & 6 & $1.49 \%$ \\
\hline African & 5 & $1.24 \%$ \\
\hline Missing data & 1 & $0.25 \%$ \\
\hline \multicolumn{3}{|l|}{ Sexuality } \\
\hline Heterosexual & 328 & $81.59 \%$ \\
\hline Bisexual & 48 & $11.94 \%$ \\
\hline Questioning & 11 & $2.74 \%$ \\
\hline Homosexual & 6 & $1.49 \%$ \\
\hline Prefer not to say & 5 & $1.24 \%$ \\
\hline Other & 4 & $1.00 \%$ \\
\hline \multicolumn{3}{|l|}{ Course } \\
\hline Semester 2, 2019 & 219 & $54.48 \%$ \\
\hline Semester 1, 2020 & 116 & $28.86 \%$ \\
\hline Semester 1, 2020 (online) & 66 & $16.42 \%$ \\
\hline Other & 1 & $0.16 \%$ \\
\hline \multicolumn{3}{|l|}{ Year of commencement } \\
\hline Semester 1, 2019 & 176 & $43.78 \%$ \\
\hline Semester 1, 2020 & 84 & $20.90 \%$ \\
\hline 2018 & 56 & $13.93 \%$ \\
\hline Semester 2, 2019 & 49 & $12.19 \%$ \\
\hline 2017 or earlier & 37 & $9.20 \%$ \\
\hline \multicolumn{3}{|l|}{ Enrolment status } \\
\hline Full-time & 344 & $85.57 \%$ \\
\hline Part-time & 58 & $14.43 \%$ \\
\hline \multicolumn{3}{|l|}{ Study mode } \\
\hline On campus & 361 & $89.80 \%$ \\
\hline Online & 41 & $10.20 \%$ \\
\hline \multicolumn{3}{|l|}{ Campus location } \\
\hline Main campus & 310 & $77.11 \%$ \\
\hline Satellite campus & 84 & $20.90 \%$ \\
\hline Other & 8 & $1.99 \%$ \\
\hline \multicolumn{3}{|l|}{ Degree } \\
\hline Science & 198 & $49.25 \%$ \\
\hline
\end{tabular}


Table 2 (continued)

\begin{tabular}{llr}
\hline Variable & $N$ & Percentage \\
\hline Education and arts & 95 & $23.63 \%$ \\
Health and medicine & 33 & $8.21 \%$ \\
Other & 76 & $18.91 \%$ \\
Diagnosed by a doctor as having a mental illness & \\
No & 229 & $56.97 \%$ \\
Yes & 159 & $39.55 \%$ \\
Prefer not to say & 14 & $3.48 \%$ \\
Sought help from a mental health professional (e.g., counsellor, & & \\
psychologist, psychiatrist) at some point & & $61.69 \%$ \\
Yes & 248 & $36.57 \%$ \\
No & 147 & $1.74 \%$ \\
Prefer not to say & 7 & $95.52 \%$ \\
English as first language & & $4.48 \%$ \\
Yes & 384 & 18 \\
No & & \\
\hline
\end{tabular}

Please note that a virus pandemic caused significant disruption to Australian residents and students during the first part of 2020, and the participating university moved to online teaching on 23rd March 2020. This event and the associated disruption may have affected participants who completed the survey during 2020. However, contrary to this possibility, the mental health of participants who completed the survey during $2020(n=116,28.86 \%)$ was not significantly different from that of participants who completed the study in 2019 ( $n=286,71.14 \%$; $p \mathrm{~s} \geq 0.276$, $\mathrm{BF}_{01} \mathrm{~s} \geq 1.96$ ). These nonsignificant results are consistent with other research that has found that the coronavirus pandemic did not have a significant effect on students' mental health during March 2020 (Fried et al. 2020).

\section{Preliminary analyses: subjective social status, mental health, and satisfaction with university}

Table 3 provides the descriptive statistics and Pearson correlation coefficients for subjective social status, mental health, satisfaction with university, and intention to stay at university.

Consistent with work that has found that university students have high levels of mental health problems (e.g., Duffy et al. 2020; Stallman 2010), the DASS mean score $(M=18.27, S D=11.78)$ was substantially higher than that for a representative sample of the general adult population of Australia $(N=497, M=8.30, S D=9.83$; Crawford et al. 2011, p. 9). The BMHI had a large positive correlation with the DASS ( $r=0.80, p<0.001)$, indicating good convergent validity between these two measures.

On average, participants reported that their mental health had improved slightly since starting university $(M=5.11, S D=40.81)$. This difference was small in size 


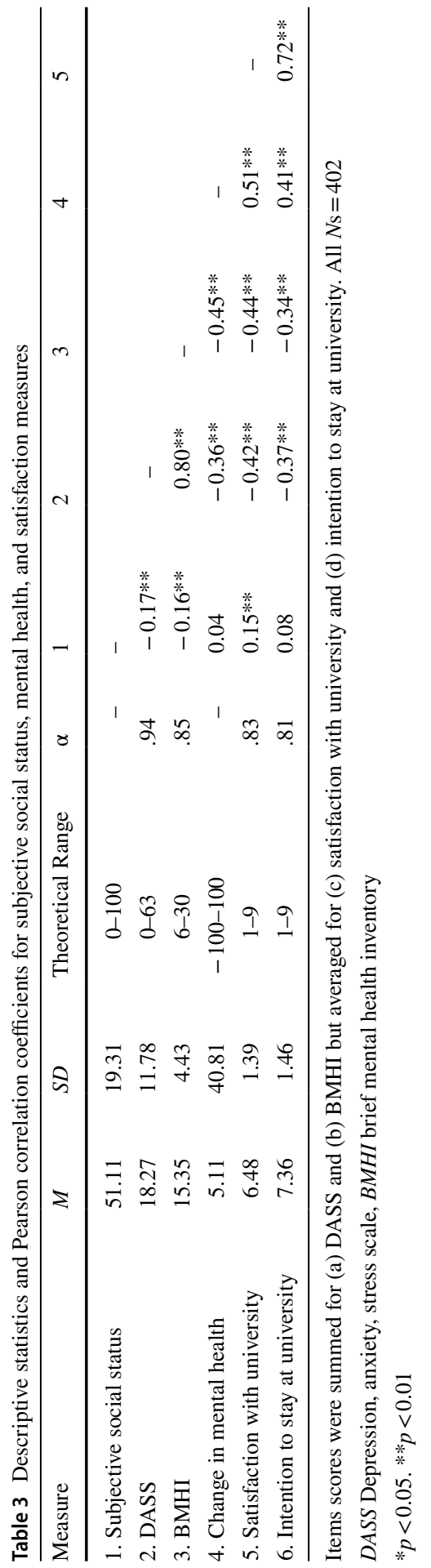


(Cohen's $d=0.13$ ), but it was significantly different from the scale's zero midpoint of no change, $t(401)=2.51, p=0.012$, although the difference represented only anecdotal evidence $\left(\mathrm{BF}_{10}=2.57\right)$. The direction of this effect is inconsistent with prior longitudinal evidence that has found that attendance at university increases depression and anxiety (Andrews and Wilding 2004).

Finally, consistent with prior work (e.g., Rubin et al. 2016), subjective social status showed significant negative correlations with DASS and BMHI, indicating that participants who reported being of higher social status also tended to report having fewer mental health problems over the past week (DASS) and the past month (BMHI). Subjective social status had no significant correlation with change in mental health $(r=0.04)$ or intention to stay at university $(r=0.08)$. However, as in prior research (Martin 2012), subjective social status was significantly positively associated with satisfaction with university $(r=0.15)$ : Participants who reported having a higher social status tended to feel more satisfied with their university experience.

Similar to Yzerbyt et al. (2018), I only considered potential mediation models if (a) the predictor was significantly related to the outcome variable, (b) the predictor was significantly related to the mediator variable, and (c) the mediator was significantly related to the outcome variable. Following these criteria, I ruled out mediations of the associations between subjective social status and (a) change in mental health and (b) intention to stay at university, because these associations were not significant. Instead, I focused on potential mediators of the associations between subjective social status and (a) DASS, (b) BMHI, and (c) satisfaction with university.

\section{Stage 1: identifying high impact issues}

Table 4 provides the means and standard deviations for each of the 32 issues that participants rated in terms of perceived degree of impact on their mental health over the past six months. The impact ratings are ranked from the most impactful to the least impactful.

Looking at Table 4, it can be seen that students' mean rating of the highest impacting issue (lack of money) only indicated "a moderate impact" on their mental health, according to the scale anchors. Hence, the following categories of "high," "medium," and "low" impact should be interpreted in this context.

In order to identify high and low impact issues, I calculated the mean of all 32 issues $(M=23.02)$ and the standard deviation of this variable $(S D=12.45)$. I then categorized issues at or above one $S D$ of this mean value (i.e., 35.47) as high impact issues and issues at or below one $S D$ of the mean (i.e., 10.57) as low impact issues.

As can be seen from Table 4, the following six issues were classed as having a relatively high impact on students' mental health: lack of money; time management; coursework assessment items; lack of sleep; course marks; formal examinations. The following 11 issues were classed as having a relatively low impact on students' mental health: homesickness; other students; eating disorder; prejudice and discrimination against me; university staff members (lecturers, tutors, etc.); managing childcare; sexual abuse/harassment; being bullied; drug use; victim of violence; sexual identity. Again, note that these relatively low ratings may have occurred because 
Table 4 Means and standard deviations of issues perceived to impact on mental health over the past six months

\begin{tabular}{|c|c|c|}
\hline & $M$ & $S D$ \\
\hline \multicolumn{3}{|l|}{ High impact issues } \\
\hline Lack of money & 45.63 & 34.65 \\
\hline Time management & 45.20 & 29.23 \\
\hline Coursework assessment items & 44.22 & 28.71 \\
\hline Lack of sleep & 43.25 & 31.61 \\
\hline Course marks & 40.19 & 31.09 \\
\hline Formal examinations & 38.65 & 31.93 \\
\hline \multicolumn{3}{|l|}{ Medium impact issues } \\
\hline Lack of exercise & 35.43 & 30.29 \\
\hline Non-university work & 33.21 & 30.20 \\
\hline Physical health & 33.02 & 30.48 \\
\hline Family members & 32.47 & 31.40 \\
\hline Loneliness & 32.05 & 32.35 \\
\hline Difficulty understanding course material & 31.95 & 27.34 \\
\hline Unhealthy diet (e.g., fast food, no fruit and veg) & 31.39 & 29.88 \\
\hline Not knowing how things work at university & 25.95 & 27.76 \\
\hline Romantic relationships & 25.62 & 28.89 \\
\hline Attending classes (lectures, tutorials, labs, etc.) & 25.40 & 25.90 \\
\hline Finding a job after university & 24.91 & 29.64 \\
\hline Lack of sense of belonging at university & 23.38 & 27.81 \\
\hline Grief & 18.18 & 27.47 \\
\hline Travelling to and from university & 17.42 & 25.78 \\
\hline Alcohol use & 11.63 & 21.61 \\
\hline \multicolumn{3}{|l|}{ Low impact issues } \\
\hline Homesickness & 10.50 & 22.05 \\
\hline Other students & 10.25 & 20.05 \\
\hline Eating disorder & 9.07 & 21.08 \\
\hline Prejudice and discrimination against me & 7.19 & 18.36 \\
\hline University staff members (lecturers, tutors, etc.) & 6.97 & 14.11 \\
\hline Managing childcare & 6.57 & 18.65 \\
\hline Sexual abuse/harassment & 5.76 & 17.73 \\
\hline Being bullied & 5.61 & 16.90 \\
\hline Drug use & 5.44 & 15.38 \\
\hline Victim of violence & 5.22 & 17.41 \\
\hline Sexual identity & 4.83 & 15.16 \\
\hline
\end{tabular}

Scores had a theoretical range of 0 to 100 . Issues are ranked by mean value in descending order. Issues categorized as high and low impact are one standard deviation above and below the overall mean for all issues combined, respectively

either (a) participants did not tend to experience these issues (i.e., the issues were not prevalent in the population), or (b) participants experienced these issues, but the issues were not perceived to have had an effect on their mental health (i.e., the issues 
were prevalent but not particularly impactful). The low prevalence explanation is the most likely in the case of issues such as homesickness, eating disorder, sexual abuse/ harassment, being bullied, and victim of violence, but this point does not invalidate the conclusion that these issues had a low impact on the mental health of the student population.

\section{Stage 2: identifying high impact issues that mediate the association between subjective social status and mental health}

Table 5 shows the correlations between subjective social status, outcome variables (mental health and satisfaction), and the six high impact issues. As can be seen from Column 1 of Table 5, subjective social status had small-to-medium sized, significant, negative correlations with all issues apart from formal examinations. The largest of these correlations was between subjective social status and lack of money $(r=-0.31, p<0.001)$. However, the size of this correlation was not sufficiently large to threaten the divergent validity of either of these two measures (i.e., it was $\operatorname{not} \geq 0.70$ ).

As shown in Columns 2-4, the three outcome variables had significant correlations with all high impact issues. Hence, similar to Yzerbyt et al.'s (2018) approach, I proceeded to consider all issues apart from formal examinations as potential mediators of the associations between subjective social status and (a) DASS, (b) BMHI, and (c) satisfaction with university.

I conducted mediation analyses using Model 4 of Hayes' (2018) PROCESS software. In this model, subjective social status was the predictor variable and

Table 5 Pearson correlation coefficients between subjective social status, outcome variables, and high impact issues

\begin{tabular}{|c|c|c|c|c|c|c|c|c|}
\hline Measure & 1 & 2 & 3 & 4 & 5 & 6 & 7 & 8 \\
\hline $\begin{array}{l}\text { 1. Subjective social } \\
\text { status }\end{array}$ & - & & & & & & & \\
\hline 2. DASS & $-0.17 * *$ & - & & & & & & \\
\hline 3. BMHI & $-0.16 * *$ & $0.80 * *$ & - & & & & & \\
\hline $\begin{array}{l}\text { 4. Satisfaction with } \\
\text { university }\end{array}$ & $0.15^{* *}$ & $-0.42 * *$ & $-0.44 * *$ & - & & & & \\
\hline 5. Lack of money & $-0.31 * *$ & $0.34 * *$ & $0.28 * *$ & $-0.14 * *$ & - & & & \\
\hline 6. Time management & $-0.15 * *$ & $0.36 * *$ & $0.33 * *$ & $-0.28 * *$ & $0.26 * *$ & - & & \\
\hline $\begin{array}{l}\text { 7. Coursework assess- } \\
\text { ment items }\end{array}$ & $-0.15 * *$ & $0.24 * *$ & $0.29 * *$ & $-0.33 * *$ & $0.31 * *$ & $0.53 * *$ & & \\
\hline 8. Lack of sleep & $-0.11 *$ & $0.36 * *$ & $0.33 * *$ & $-0.13 * *$ & $0.32 * *$ & $0.41 * *$ & $0.34 * *$ & \\
\hline 9. Course marks & $-0.16^{* *}$ & $0.18 * *$ & $0.21 * *$ & $-0.20 * *$ & $0.30 * *$ & $0.42 * *$ & $0.62 * *$ & $0.33 * *$ \\
\hline $\begin{array}{l}\text { 10. Formal examina- } \\
\text { tions }\end{array}$ & -0.08 & $0.19 * *$ & $0.23 * *$ & $-0.17 * *$ & $0.26 * *$ & $0.39 * *$ & $0.49 * *$ & $0.26 * *$ \\
\hline
\end{tabular}

All Ns $=402$. DASS Depression, anxiety, stress scale, $B M H I$ brief mental health inventory

$* p<0.05$. ** $p<0.01$ 
DASS, BMHI, or satisfaction with university were the outcome variables. Lack of money, time management, coursework assessment items, lack of sleep, and course marks were the mediator variables. I used 5000 bootstrapping iterations and bias-corrected bootstrapped $95 \%$ confidence intervals to test the significance of indirect effects.

Preliminary results showed that each of the five high impact issues mediated the associations between subjective social status and (a) DASS, (b), BMHI, and (c) satisfaction with university, $\beta \mathrm{s} \geq 0.013, p \mathrm{~s} \leq 0.05$. However, the mental health measures had medium-sized negative correlations with the measure of satisfaction with university ( $r \mathrm{~s} \geq 1.42 \mathrm{l})$. Again, this result is consistent with prior evidence (e.g., Almhdawi et al. 2018; Karaca et al. 2019), and it suggests that some of the initial mediation effects may have occurred due to shared variance between mental health and satisfaction with university. In order to provide more diagnostic tests, I included satisfaction with university as a covariate when testing mediators in relation to DASS and BMHI, and I included DASS and BMHI as covariates when testing mediators in relation to satisfaction with university. I tested the indirect effects of each issue (a) separately and (b) together with the other four issues in parallel. The parallel mediation analysis tests the indirect effect of each mediator variable while controlling for the indirect effects of the other mediator variables. Hence, it provides a method of testing the independent mediating effect of each mediator. Table 6 provides the results from these analyses.

As can be seen in Table 6, lack of money consistently and independently mediated the association between subjective social status and mental health (DASS \& BMHI). Time management also mediated this effect, although less consistently, because it was not significant in relation to the BMHI in the parallel mediation analysis. The other consistent finding was that coursework assessment items mediated the association between subjective social status and satisfaction with university. Course marks also mediated this effect. Again, however, course marks was not a significant mediator in the parallel analysis.

The completely standardised indirect effects for the above effects ranged in size from 0.022 to -0.084 . For mediation effects, a small effect $=0.010$, a medium effect $=0.090$, and a large effect $=0.250$ (Kenny 2018). Hence, these indirect effects were small-to-medium in size.

I repeated Table 6's mediation analyses but included PARH scores as a covariate in order to check whether participants' perceived awareness of putative research hypotheses had any impact on the pattern of significant and nonsignificant mediation effects (Rubin 2016). The pattern of results was almost identical. The exception was that the mediating effect of coursework assessment items in relation to BMHI was reduced to nonsignificance in the simple mediation analyses. Hence, the reported results do not appear to be influenced by demand characteristics.

I also repeated Table 6's mediation analyses after excluding outliers. Outliers were defined as scores that were $\pm 3.00 S D$ s from sample means. There were 3 outliers on the DASS, 1 on the BMHI, and 2 on satisfaction with university. The pattern of significant and nonsignificant results remained the same when these outliers were excluded for each associated outcome variable. 


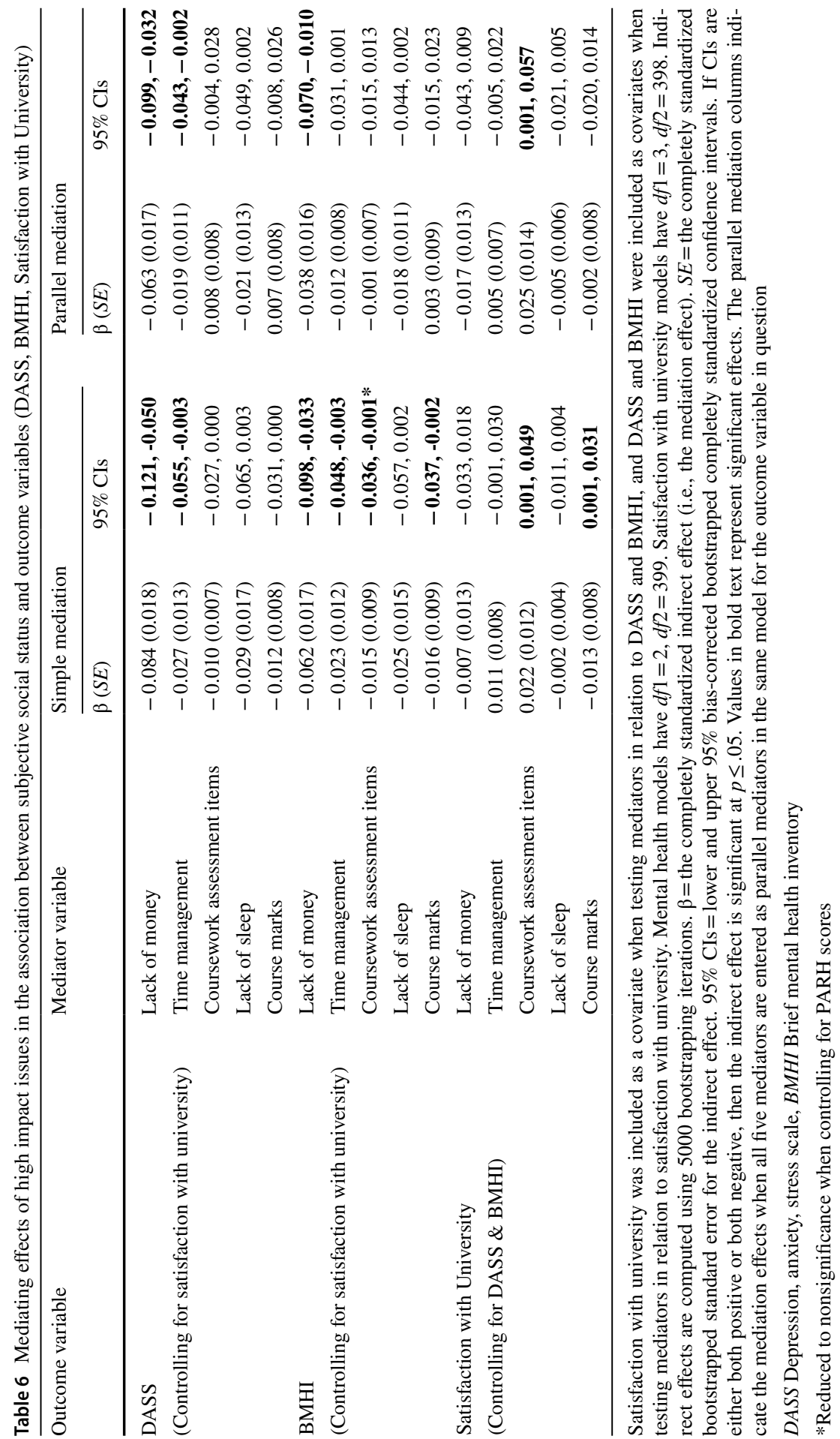




\section{Discussion}

The first-year psychology undergraduate students who took part in this study rated lack of money, time management, coursework assessment items, lack of sleep, course marks, and formal examinations as having a relatively high impact on their mental health over the past six months. Interestingly, students rated "prejudice and discrimination against me" as having a relatively low impact on their mental health, and subjective social status did not have a significant correlation with this variable $(r=-0.09, p=0.064)$. Hence, contrary to Allan et al. (2016), classism did not appear to be a major issue, at least among the first-year undergraduate psychology students that I sampled, although it should be noted that the reliability and validity of this single item measure can be questioned. Similarly, although there was a significant negative correlation between subjective social status and the perceived mental health impacts of (a) loneliness $(r-0.13, p=0.008)$ and (b) lack of sense of belonging at university $(r=-0.10, p=0.043)$, students rated these issues as having only a medium impact on their mental health. Hence, although Rubin et al.'s (2016) social contact explanation received some support, students did not perceive a strong link between lack of social contact with university friends and their mental health. These results do not deny that classism and lack of social contact mediate the association between subjective social status and mental health. However, they do suggest that interventions based on these issues may face an uphill struggle in motivating students to take these issues seriously.

The present research was able to identify mediators of the association between subjective social status and mental health from among a group of issues that had received relatively high impact ratings. In particular, the perceived impact of lack of money and, less consistently, time management mediated the association between subjective social status and mental health while controlling for satisfaction with university. These results are consistent with prior work that has shown that lack of money and time explain the disadvantages experienced by students from lower SES backgrounds in other areas of university life, namely social integration (Rubin and Wright 2017). The current results build on this prior work by showing that a similar mediation effect applies to the association between subjective social status and mental health. Students who reported having a lower social status rated lack of money and time management as having a relatively large impact on their mental health, and these ratings helped to explain their poorer mental health (DASS \& BMHI). These results make sense because money and time are resources that can be used to buffer the impact of stressful events on mental health. For example, students with more money can afford to pay for stress-relieving activities, such as social activities (e.g., Cabrera et al., 1992; Martin 2012). In addition, students with better time management opportunities can complete their university studies in a less stressful manner (Macan et al. 1990).

Interestingly, although students who reported having less subjective social status believed that their coursework assessment items and course marks had a relatively high negative impact on their mental health, this belief did not tend to explain (mediate) their poorer mental health. It only explained their poorer satisfaction with 
their university experience. In particular, perceived impact of coursework assessment items and, less consistently, course marks mediated the association between subjective social status and satisfaction with university. These results are consistent with prior research that has shown that students from lower SES backgrounds tend to be less satisfied with their university experience than students from higher SES backgrounds (Martin 2012), and that course marks are positively associated with satisfaction with university (for a review, see Green et al. 2015, pp. 139-140).

\section{Limitations}

The present results refer to a measure of subjective social status. It is unclear whether they extend to more objective measures of social class. Having said that, there are good theoretical and empirical grounds for expecting a close connection between these subjective and objective measures (Adler et al. 2000), including in university samples (Kraus et al. 2011; Rubin and Stuart 2018; Rubin et al. 2014), and so I predict that the current results will replicate using more objective measures of social class.

It is also important to appreciate that the impacting issues in the present research confounded the frequency with which students experienced potentially impacting issues with the perceived impact of those issues on their mental health. For example, participants in this study may have rated being the victim of violence as having a relatively low impact on their mental health over the past six months because either (a) they had not experienced being the victim of violence during this time period or (b) they had experienced being the victim of violence but it did not have a large impact on their mental health. It is important to appreciate that this confound between frequency of experience and perceived impact does not undermine the conclusions of the current study, because these conclusions refer to the student population rather than to individual students. Hence, it remains valid to claim that issues such as drug use, being a victim of violence, and sexual identity had a relative low impact on the mental health of students, even if the reason for this low impact is the relatively low frequency with which students experienced these issues. It is also worth noting that the current approach is consistent with prior work by Juniper et al. (2012), who measured experience (frequency) and subjective importance separately, but then created an impact score based on the product of frequency and importance. This approach is conceptually similar to the single scale approach that was used in the present study. Nonetheless, further research is required to confirm that (a) lack of money and time management mediate the positive association between social class and mental health, and (b) coursework assessment items and course marks mediate the positive association between subjective social class and satisfaction with university.

The present research used a cross-sectional correlational design. Hence, no firm conclusions can be made regarding the causal direction of the associations. For example, it is possible that poorer mental health led students to rate themselves lower on the measure of subjective social status. 
Finally, it is important to acknowledge that the current sample consisted of $83.83 \%$ women. This gender imbalance is typical for psychology degrees, and the current sample was drawn from psychology courses. Hence, the sample is representative in this sense. Women sometimes report slightly more mental health problems than men (e.g., Crawford et al. 2011, p. 9). However, this gender difference was not significant in the present sample $(p s \geq 0.112)$. An exploratory investigation of gender differences in impact ratings found that, compared to men, women rated the following issues as being more impactful: lack of money, physical health, difficulty understanding course material, family members, eating disorder, sexual abuse/harassment, homesickness, travelling to and from university, unhealthy diet, not knowing how things work at university, and lack of exercise ( $p s \leq 0.041)$. Lack of money is the only variable in this list that was used as a mediator variable in the present analyses. Consequently, I repeated the simple mediation analyses that included lack of money, but this time I included gender (coded as male, female, or missing data) as a covariate. The pattern of significant and nonsignificant indirect effects that are reported in Table 6 remained the same. Hence, gender did not appear to affect the results in this respect.

\section{Implications}

The present research provides a novel approach to understanding mental health among university students by obtaining an empirical assessment of the perceived impact of issues that may affect metal health. Contrary to prior impact analyses with university students (Juniper et al. 2012), the present research used a single scale measure of impact and then considered the high impact issues as potential mediators of the association between subjective social status and mental health among firstyear psychology undergraduate students.

Based on the current results, students rated lack of money and time management as being most detrimental to their mental health, and they rated coursework assessment and course marks as being most detrimental to satisfaction with their university experience. Hence, students should not need a greater deal of persuading that these issues are problematic, and interventions that focus on these issues are likely to be more successful in terms of student uptake. For example, interventions might take the form of financial subsidies, time management training, and the use of less stressful coursework assessments.

Of course, there is a balance to be struck between the student uptake of an intervention and its theoretical efficacy. Ideally, we should strive for interventions that have both large effects and good uptake. It is obviously not useful to focus on interventions that have negligible effects, even if their uptake is very good. Similarly, it is problematic to focus on interventions that have massive effects but almost no uptake. Most research in the current area has focused on effect sizes rather than student uptake. For example, Rubin et al. (2016, p. 734) explained that, when extrapolating to the population level, their small-to-medium sized effects had "the potential to affect thousands of students in higher education." The present research builds 
on this point by focusing on the potential for student uptake and considering which putative processes are more likely to be accepted by students and acted on.

Of course, it is not difficult to persuade students of the need for greater financial subsidising of their university education. However, the present research findings indicate that more students may be willing to accept that financial subsidies will help to reduce social class disparities in mental health relative to interventions that address issues such as drug use, violence, or sexual identity, because more students will have personal experience of lack of money affecting their mental health. To be clear, these latter issues are vitally important, and they deserve funding towards their reduction in student populations. However, the present research findings suggest that most students are unlikely to accept that interventions that address drug use, being a victim of violence, and sexual identity will lead to significant improvements in their own mental health, because most students do not view these issues as being impactful on their own mental health.

Providing greater training and opportunity for effective time management may also be beneficial to students from lower SES backgrounds. It is possible that these students need to manage a greater number of extracurricular activities, such as paid work, travel, and childcare. However, contrary to this possibility, subjective social status was not significantly associated with the impact ratings for non-university work $(r=-0.08, p=0.105)$ or travelling to and from university $(r=-0.08$, $p=0.114$ ), and it was only weakly negatively associated with the impact ratings for managing childcare $(r=-0.11, p=0.034)$. Nonetheless, better time management skills may facilitate students from lower SES backgrounds in managing their busy lives.

Finally, the present research findings suggest that reducing the number of coursework assessment items and placing less weight on course marks may help to reduce social class differences in satisfaction with the university experience. In practice, these issues need to be thought through carefully because they are inversely related. For instance, reducing the number of coursework assessment items may result in fewer items that each have relatively larger course weightings (e.g., two items each worth $50 \%$ of the final course mark rather than five items each worth $20 \%$ ). In this case, a reduction in coursework assessment items may actually increase the impact of each coursework item on mental health. A more effective approach to this issue may be to reduce the number of stressful coursework items. This may be achieved by providing clearer assessment instructions, more flexible arrangements for awarding extended due dates, practice tests, more positive feedback, greater emphasis on learning rather than achievement, and so on.

\section{Conclusion}

The students who took part in this study rated lack of money, time management, coursework assessment items, lack of sleep, and course marks as having the largest impact on their mental health. Furthermore, lack of money and time management mediated the positive association between subjective social status and mental health, and coursework assessment items and course marks mediated the positive 
association between subjective social status and satisfaction with the university experience. It is concluded that interventions should focus on these relatively high impact issues in order to address social class differences in mental health and university satisfaction.

Data availability The preregistered research protocol, research materials, dataset, and data aggregation code associated with the current study are available in the Open Science Framework repository at https:// osf.io/ejycf/

\section{Compliance with ethical standards}

Conflict of interest The author declares that they have no conflict of interest.

\section{References}

Adler NE, Epel ES, Castellazzo G, Ickovics JR (2000) Relationship of subjective and objective social status with psychological and physiological functioning: preliminary data in healthy, White women. Health Psychol 19:586-592. https://doi.org/10.1037/0278-6133.19.6.586

Allan BA, Garriott PO, Keene CN (2016) Outcomes of social class and classism in first-and continuinggeneration college students. J Couns Psychol 63(4):487-496. https://doi.org/10.1037/cou0000160

Almhdawi KA, Kanaan SF, Khader Y, Al-Hourani Z, Almomani F, Nazzal M (2018) Study-related mental health symptoms and their correlates among allied health professions students. Work 61(3):391-401. https://doi.org/10.3233/WOR-182815

Andrews B, Wilding JM (2004) The relation of depression and anxiety to life-stress and achievement in students. Br J Psychol 95:509-521. https://doi.org/10.1348/0007126042369802

Berwick DM, Murphy JM, Goldman PA, Ware JE, Barsky AJ, Weinstein MC (1991) Performance of a fiveitem mental health screening test. Med Care 29:169-176. https://doi.org/10.1097/00005650-19910 2000-00008

Cabrera AF, Nora A, Castaneda MB (1992) The role of finances in the persistence process: a structural model. Res High Educ 33(5):571-593. https://doi.org/10.1007/BF00973759

Crawford J, Cayley C, Lovibond PF, Wilson PH, Hartley C (2011) Percentile norms and accompanying interval estimates from an Australian general adult population sample for self-report mood scales (BAI, BDI, CRSD, CES-D, DASS, DASS-21, STAI-X, STAI-Y, SRDS, and SRAS). Aust Psychol 46(1):314. https://doi.org/10.1111/j.1742-9544.2010.00003.x

Diemer MA, Mistry RS, Wadsworth ME, López I, Reimers F (2013) Best practices in conceptualizing and measuring social class in psychological research. Anal Soc Issues Public Policy 13(1):77-113. https:// doi.org/10.1111/asap.12001

Duffy A, Keown-Stoneman C, Goodday S, Horrocks J, Lowe M, King N, Bisdounis L et al (2020) Predictors of mental health and academic outcomes in first-year university students: Identifying prevention and early-intervention targets. BJPsych Open. https://doi.org/10.1192/bjo.2020.24

Faul F, Erdfelder E, Lang AG, Buchner A (2007) G*Power 3: a flexible statistical power analysis program for the social, behavioral, and biomedical sciences. Behav Res Methods 39(175):191. https://doi. org/10.3758/BF03193146

Fried E, Papanikolaou F, Epskamp S (2020) Mental health and social contact during the covid-19 pandemic: an ecological momentary assessment study. https://osf.io/mvdpe/

Funder, D. C., \& Ozer, D. J (2019) Evaluating effect size in psychological research: sense and nonsense. Adv Methods Pract Psychol Sci 2(2):156-168. https://doi.org/10.1177/2515245919847202

Green HJ, Hood M, Neumann DL (2015) Predictors of student satisfaction with university psychology courses: a review. Psychol Learn Teach 14(2):131-146. https://doi.org/10.1177/1475725715590959

Hayes AF (2018) Introduction to mediation, moderation, and conditional process analysis: a regression-based approach, 2nd edn. Guildford Press, New York

Henry JD, Crawford JR (2005) The short-form version of the depression anxiety stress scales (DASS-21): construct validity and normative data in a large non-clinical sample. Br J Clin Psychol 44(2):227-239. https://doi.org/10.1348/014466505x29657 
Ibrahim AK, Kelly SJ, Glazebrook C (2013) Socioeconomic status and the risk of depression among UK higher education students. Soc Psychiatry Psychiatr Epidemiol 48(9):1491-1501. https://doi. org/10.1007/s00127-013-0663-5

Juniper B, Walsh E, Richardson A, Morley B (2012) A new approach to evaluating the well-being of $\mathrm{PhD}$ research students. Assess Eval High Educ 37(5):563-576. https://doi.org/10.1080/02602 938.2011.555816

Karaca A, Yildirim N, Cangur S, Acikgoz F, Akkus D (2019) Relationship between mental health of nursing students and coping, self-esteem and social support. Nurse Educ Today 76:44-50. https://doi. org/10.1016/j.nedt.2019.01.029

Kenny D (2018) Mediation. https://davidakenny.net/cm/mediate.htm

King S, Garrett R, Wrench A, Lewis N (2011) The loneliness of relocating: does the transition to university pose a significant health risk for rural and isolated students. Paper presented at the First Year in Higher Education Conference, Brisbane, Australia. Retrieved from https://fyhe.com.au/past_papers/papers11/ FYHE-2011/content/pdf/16B.pdf

Kraus MW, Piff PK, Keltner D (2011) Social class as culture: the convergence of resources and rank in the social realm. Curr Dir Psychol Sci 20(4):246-250. https://doi.org/10.1177/0963721411414654

Lorant V, Deliège D, Eaton W, Robert A, Philippot P, Ansseau M (2003) Socioeconomic inequalities in depression: a meta-analysis. Am J Epidemiol 157(2):98-112. https://doi.org/10.1093/aje/kwf182

Macan TH, Shahani C, Dipboye RL, Phillips AP (1990) College students' time management: correlations with academic performance and stress. J Educ Psychol 82(4):760-768. https://doi. org/10.1037/0022-0663.82.4.760

Martin ND (2012) The privilege of ease: Social class and campus life at highly selective, private universities. Res High Educ 53(4):426-452. https://doi.org/10.1007/s11162-011-9234-3

Rubin M (2016) The perceived awareness of the research hypothesis scale: assessing the influence of demand characteristics. Figshare. https://doi.org/10.6084/m9.figshare.4315778

Rubin M, Denson N, Kilpatrick S, Matthews KE, Stehlik T, Zyngier D (2014) “I am working-class:” subjective self-definition as a missing measure of social class and socioeconomic status in higher education research. Educ Res 43(4):196-200. https://doi.org/10.3102/0013189X14528373

Rubin M, Evans O, McGuffog R (2019) Social class differences in social integration at university: Implications for academic outcomes and mental health. In: Jetten J, Peters K (eds) The social psychology of inequality. Springer, Cham, pp 87-102

Rubin M, Evans O, Wilkinson RB (2016) A longitudinal study of the relations between university students' subjective social status, social contact with university friends, and mental health and well-being. J Soc Clin Psychol 35:722-737. https://doi.org/10.1521/jscp.2016.35.9.722

Rubin M, Kelly BM (2015) A cross-sectional investigation of parenting style and friendship as mediators of the relation between social class and mental health in a university community. Int J Equity Health 14(87):1-11. https://doi.org/10.1186/s12939-015-0227-2

Rubin M, Stuart R (2018) Kill or cure? Different types of social class identification amplify and buffer the relation between social class and mental health. J Soc Psychol 158(2):236-251. https://doi. org/10.1080/00224545.2017.1327405

Rubin M, Wright CL (2017) Time and money explain social class differences in students' social integration at university. Stud High Educ 42:315-330. https://doi.org/10.1080/03075079.2015.1045481

Said D, Kypri K, Bowman J (2013) Risk factors for mental disorder among university students in Australia: findings from a web-based cross-sectional survey. Soc Psychiatry Psychiatr Epidemiol 48:935-944. https://doi.org/10.1007/s00127-012-0574-x

Schoemann AM, Boulton AJ, Short SD (2017) Determining power and sample size for simple and complex mediation models. Soc Psychol Pers Sci 8:379-386. https://doi.org/10.1177/1948550617715068

Stallman HM (2010) Psychological distress in university students: a comparison with general population data. Aust Psychol 45:249-257. https://doi.org/10.1080/00050067.2010.482109

Yzerbyt VY, Muller D, Batailler C, Judd CM (2018) New recommendations for testing indirect effects in mediational models: the need to report and test component paths. J Pers Soc Psychol. https://doi. org/10.1037/pspa0000132

Publisher's Note Springer Nature remains neutral with regard to jurisdictional claims in published maps and institutional affiliations. 